\author{
D) Check for updates \\ Cite this: Green Chem., 2021, 23, \\ 2317 \\ Received 18th December 2020, \\ Accepted 11th February 2021 \\ DOI: $10.1039 / \mathrm{dOgc04282j}$ \\ rsc.li/greenchem
}

\section{One-step method for the preparation of cationic nanocellulose in reactive eutectic media $\uparrow$}

\author{
Esther E. Jaekel, (D) a Juho Antti Sirviö, (D) ${ }^{\mathrm{b}}$ Markus Antonietti (D) a and \\ Svitlana Filonenko (iD *a
}

\begin{abstract}
A novel method based on the use of reactive eutectic media for the extraction of cellulose nanocrystals and their functionalization with positively charged functional groups in a one-step procedure is presented. With its ability to form strong hydrogen bonds, the eutectic medium serves as a cellulose-solubilizing agent, while the presence of a Leuckart reagent provides the reactivity toward the reductive amination of carbonyl groups in the cellulose chains. In the proposed method, amorphous cellulose domains are partially dissolved by undergoing aminolysis, resulting in the formation of water-dispersible nanocellulose products with high crystallinity.
\end{abstract}

\section{Introduction}

As one of the main components of lignocellulosic biomass, cellulose is the most abundant biopolymer on earth and is available to us as an almost inexhaustible resource due to the continuous regrowth of plants. While it has served as a pivotal source of energy and as construction or clothing material throughout human history, research of the last decades has discovered cellulose as the source of a unique biocompatible crystalline nanomaterial with remarkable mechanical properties. ${ }^{1}$ Extraction of those desirable nanocrystals from plant cellulose fibers is based on the selective acidic hydrolysis of the amorphous regions, which periodically alternate with the larger crystalline domains. ${ }^{2,3}$ The obtained rod-like nanoparticles or whiskers are commonly referred to as cellulose nanocrystals (CNC). The combination of advantageous properties and sustainable origin makes CNCs one of the most

\footnotetext{
${ }^{a}$ Max Planck Institute of Colloids and Interfaces, Am Mühlenberg 1, 14476 Potsdam, Germany.E-mail: svitlana.filonenko@mpikg.mpg.de

${ }^{b}$ Fibre and Particle Engineering Research Unit, University of Oulu, P.O. Box 4300, 90014 Oulu, Finland

$\dagger$ Electronic supplementary information (ESI) available. See DOI: 10.1039/ doge04282j
}

promising material building blocks of the future. ${ }^{4}$ However, a safer and economically reasonable large-scale CNC production requires alternatives to the commonly applied hydrolysis with strong inorganic acids, although attempts have been made to increase recyclability of the used acid. ${ }^{5}$

Among the alternative extraction methods, the utilization of ionic media with the ability to form strong hydrogen bonds deserves special attention: ${ }^{6}$ Deep eutectic solvents (DES) are simple to prepare and are made of low toxic (or even nontoxic) components, often derived from renewable resources. ${ }^{7,8}$ In combination with their low melting temperature, DES provide optimal conditions to process natural carbohydrate polymers in a sustainable fashion. ${ }^{9}$ They have already proven effective in wood treatment, lignocellulose delignification, and nanocellulose extraction and modification. ${ }^{10-13}$

The treatment of cellulose in eutectic media facilitates the subsequent mechanical disintegration due to partial dissolution of the hemicellulose fraction and swelling of the cellulose fibers. ${ }^{10,11}$ This effect is enhanced using derivatizing or reactive eutectic media (REM), which cause a chemical modification of the carbohydrates. Thus, charged functionalities can be introduced to the cellulose surface, decreasing the mechanical energy required for the disintegration into nanoscale fibers or particles by electrostatic repulsion. ${ }^{14}$ Like conventional TEMPO or acid treatments, the reported DES treatments most often introduce anionic functionalities into the CNC through carboxylation. ${ }^{15-19}$ Few treatments are reported to introduce cationic groups, such as guanidine, and they usually require multiple processing steps. ${ }^{20-23} \mathrm{~A}$ cheap and facile path to cationic nanocellulose however broadens their application range, for example as sorbents for wastewater treatment to remove harmful anionic contaminants, ${ }^{23,24}$ and expands the toolbox of building blocks for complex and sustainable composite materials. ${ }^{25}$

This work aims to meet the requirements of green chemistry to prepare high-value materials from sustainable resources, using technologies and chemicals with minimized 
impact on the environment. With the proposed method, CNCs are extracted and functionalized with cationic groups in a sustainable one-step procedure, using abundant and nontoxic natural chemicals.

\section{Results and discussion}

Eutectic mixtures offer a broad range of design possibilities, and their reactivity, as a function of their composition, can be tuned by thoughtful selection of the components. Our previous research revealed increased activity of ammonium formate towards the reductive amination of different substrates, ${ }^{26}$ particularly carbohydrates, ${ }^{27}$ when applied as a component of a eutectic medium, which in this case becomes reactive. Ammonium formate is the salt of ammonia with formic acid, two of the cheapest bulk chemicals.

Based on those findings, REMs containing ammonium formate $\left(T_{\mathrm{m}} 114^{\circ} \mathrm{C}\right)$ as the main hydrogen bond acceptor component were prepared in combination with different organic acids, namely glycolic, lactic, and levulinic acid, used as hydrogen bond donors and effective melting point depressors (Table S $3 \dagger$ ). When mixing the components, the REM transforms into a colorless transparent liquid, indicating the formation of a eutectic. Differential scanning calorimetry (DSC) of the used REM confirms the formation of the liquid phase at a temperature below the melting temperature of the components. Within the applied temperature range $\left(-80-50{ }^{\circ} \mathrm{C}\right)$, the REM remains thermally stable throughout three heating/ cooling cycles (Fig. S11†).

The efficacy of the REM treatment was tested in a static reactor using different REM compositions and reaction conditions (Table S3†). Pure short fiber cellulose (SFC) and de-lignified softwood kraft pulp (cellulose content 86\%) served as starting materials. Browning of the reaction mixture indicates the formation of Maillard products. This implies partial cleavage of the polysaccharides into oligo- or monosaccharides via aminolysis. X-ray diffraction (XRD) measurements of the separ- ated and purified solid products verify an increase of the crystallinity index (CrI) by 2-7\% compared to the starting material SFC, proving the dissolution of the more susceptible amorphous cellulose sections in all of the tested REMs, while crystalline regions remain intact.

The REM treatment results in a considerably reduced fiber length and fiber separation, as observed with polarized light microscopy (see Fig. S2 $\dagger$ ). An aqueous dispersion of polydisperse cellulose particles with restricted sedimentation stability is obtained: The sediment consists of larger fibers and surface adsorbed nanoparticles with lower charge, while electrostatically stabilized nanoparticles form a supernatant colloidal suspension that is stable for several months. These particles exhibit a positive zeta potential in the range of $21-36 \mathrm{mV}$. Impedance and laser diffraction measurements determine the size of particles separated by sedimentation to approximately 5-30 $\mu \mathrm{m}$, while the stable colloidal fraction showed an average size of $321 \mathrm{~nm}$. Electron microscopy imaging (Fig. 1) reveals nanoscaled cellulose whiskers about 100-300 nm in length and $c a .10 \mathrm{~nm}$ in width, which is in the usual range for CNCs from wood pulp. ${ }^{28}$ The degree of polymerization $\left(D_{\mathrm{P}}\right)$ of the anhydroglucose chains, as estimated viscometrically, decreases significantly from 2300 in the pristine SFC to around 300-330 in the particles. This corresponds to an average chain length of 155-170 nm, which is in good accordance with the particle size observed via TEM and is in the expected range (140-200 for crystalline domains in bleached wood pulp ${ }^{29}$ ). GPC analysis of the product, which was previously derivatized via carbanilation to make it soluble in DMSO, exhibits a large fraction of molecules in the $M_{\mathrm{w}}$ range of 10000-1000000 Da, corresponding to CNC-composing chains on the nanoscale. A smaller shoulder in the range of 1000-10000 Da corresponds to the $M_{\mathrm{w}}$ of oligosaccharides, supposedly adsorbed at the crystal surface. ${ }^{30}$ Pulp extraction products contain a considerable fraction of larger chains with a $D_{\mathrm{P}}$ around 2000-20000 $\left(M_{\mathrm{w}}\right.$ of $\left.1000000 \mathrm{Da}-10000000 \mathrm{Da}\right)$, related to the presence of larger fibers due to the diverse nature of the starting material (see Fig. S3†).
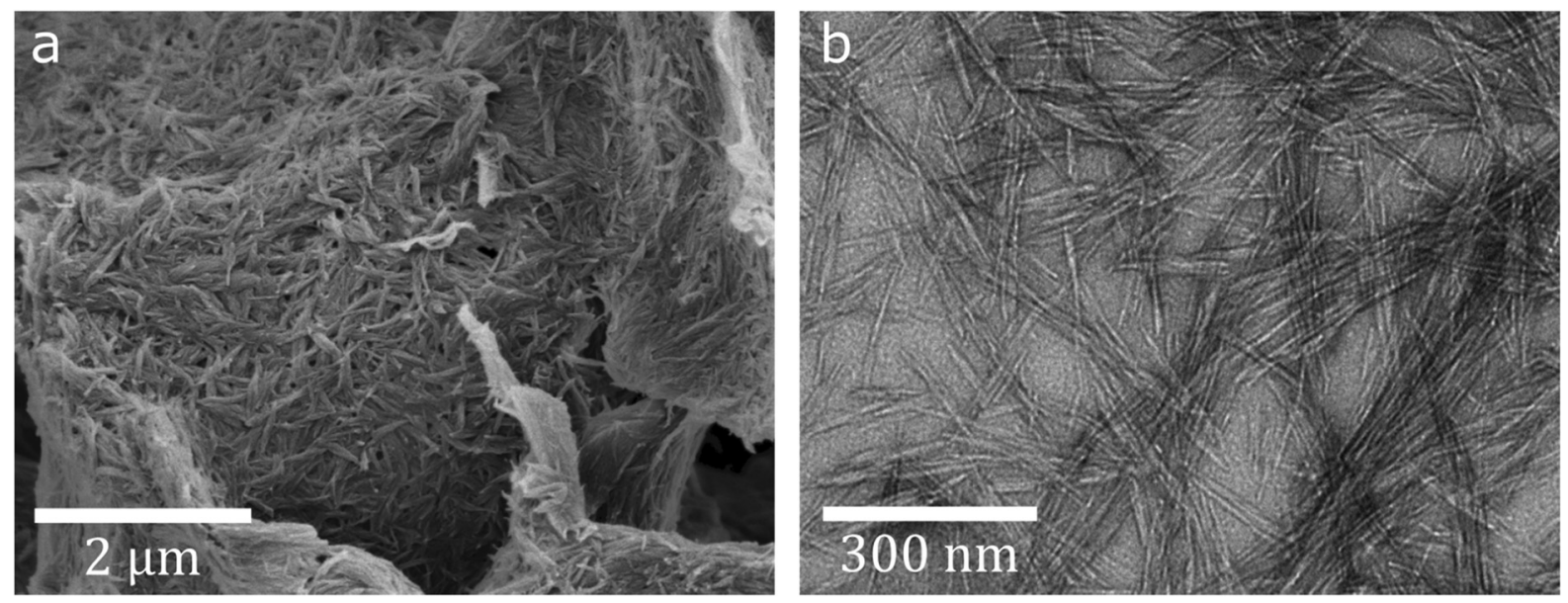

Fig. 1 (a) SEM and (b) TEM image of CNC extracted from SFC. 
Elemental analysis reveals a nitrogen content of around 0.3-0.4 wt\% in the treated cellulose. A positive ninhydrin probe (see Fig. S10†) confirms the presence of nitrogen in the form of amino groups, which, due to their basic properties, cause the positive charge in the aqueous CNC dispersion. The FTIR spectra of the extracted material and the starting material SFC do not differ substantially, proving that no severe modification of the cellulose took place, and the pristine structure of the cellulose chains is preserved (see Fig. S7 $\dagger$ ). The vibrational mode at $1640 \mathrm{~cm}^{-1}$, indicative of $\mathrm{N}-\mathrm{H}$ bending in primary amino groups, is detected with weak intensity, in accordance with the low degree of amination (Fig. 2). More conclusive difference spectra of the treated samples and the pristine cellulose (see Fig. S8 $\dagger$ ) show $\mathrm{NH}_{2}$-related bands at $3335 \mathrm{~cm}^{-1}$ and $3280 \mathrm{~cm}^{-1}$ (N-H stretch in primary amines), $1652 \mathrm{~cm}^{-1}(\mathrm{~N}-\mathrm{H}$ bend in primary amines), $1058 \mathrm{~cm}^{-1}$ (C-N stretch in aliphatic amines), and $667 \mathrm{~cm}^{-1}$ (N-H wag in primary amines)..$^{31,32}$

${ }^{13} \mathrm{C}$ CP/MAS (Fig. S12 $\dagger$ ) and ${ }^{1} \mathrm{H}$ MAS NMR (Fig. S13†) show that the REM-treated cellulose product has the same crystalline allomorph as pristine pulp, and confirm that no additional carbon-containing functional groups were introduced. Signals imputable to amino group hydrogens as well as $\mathrm{N}$-bound carbons in ${ }^{1} \mathrm{H}$ MAS or ${ }^{13} \mathrm{C} \mathrm{CP} / \mathrm{MAS}$ NMR respectively are not distinguishable on spectra at the obtained resolution due to expected overlap with other signals and the low degree of substitution. $^{33,34}$ X-ray photoelectron spectroscopy (XPS, Fig. S14 $\dagger$ ) of the REM-treated material detects nitrogen with a binding energy corresponding to amines or amides.
Based on the evidence, we presume that the treatment of cellulose in the reported REM has a twofold effect: Firstly, the eutectic dissolves amorphous cellulose domains by aminolysis, induced by ammonium and organic acid. Secondly, the Leuckart reagent causes derivatization by introducing amino groups into the crystalline cellulose, thus evolving electrostatically stabilized, nanocrystalline particles.

In the assumed reductive amination (Leuckart-Wallach reaction), ammonium formate attacks carbonyl sites (Scheme 1). In the absence of an oxidative pretreatment of the cellulose, which would increase the number of carbonyl sites, the only available carbonyl sites should be the reducing aldehyde ends of the cellulose chains.

According to the $D_{\mathrm{P}}$ (approx. 300) the reducing ends only make up $0.33 \%$ of the polymer. However, calculated from elemental analysis, around $4-5 \%$ of the anhydroglucose units should contain an amino group, which exceeds the percentage of reducing end groups by one order of magnitude. This implies the presence of more carbonyl sites due to intrinsic "defects", i.e. oxidized functionalities in the cellulose starting material, which are commonly introduced during the pulping process. $^{35}$ These additional reaction sites allow functionalization along the entire length of the fibers.

It was previously reported that acid hydrolysis treatment produces a material of inferior thermal stability, as it is expected that an increased ratio of reducing ends in CNC with lower DP promotes thermal degradation or depolymerization, supposedly through dehydration, which in turn causes
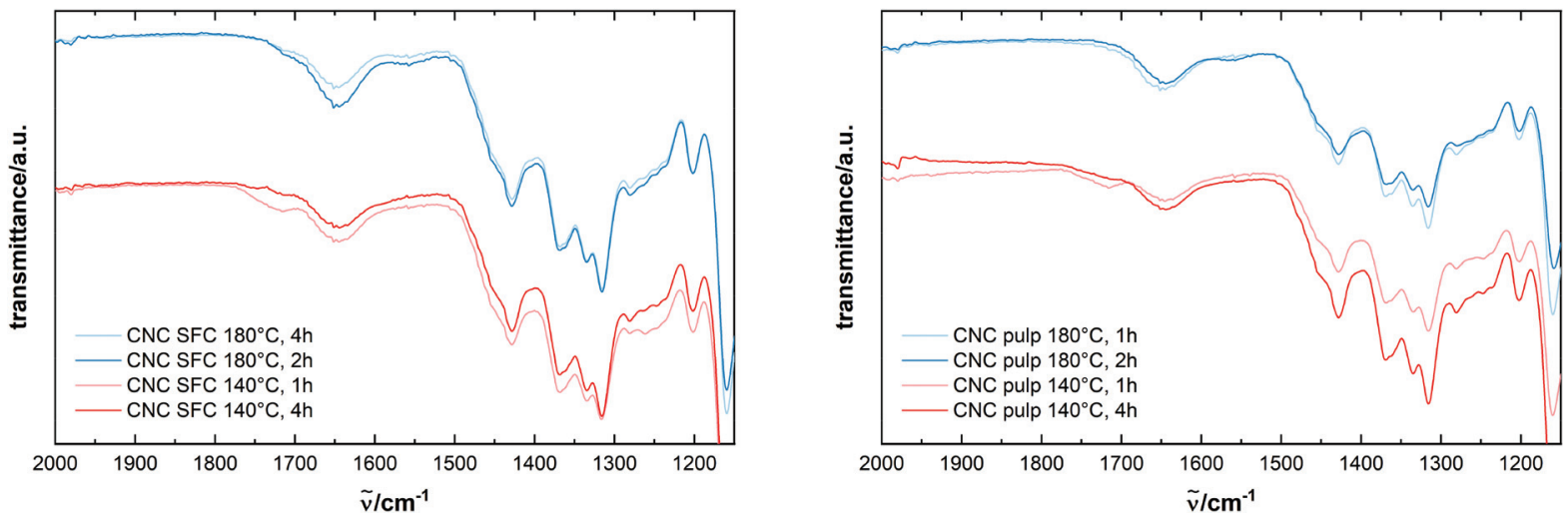

Fig. 2 ATR-IR spectra of cellulose products from the treatment of SFC with REM of ammonium formate and glycolic acid in a ratio of $2: 1$ in different conditions.
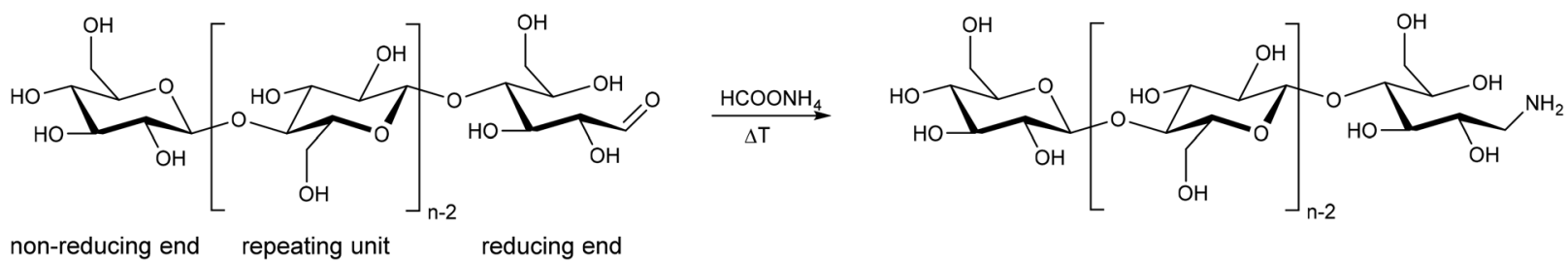

Scheme 1 Reductive amination (Leuckart-Wallach reaction) of the reducing end of a cellulose polymer chain with ammonium formate at elevated temperatures. 
(a)

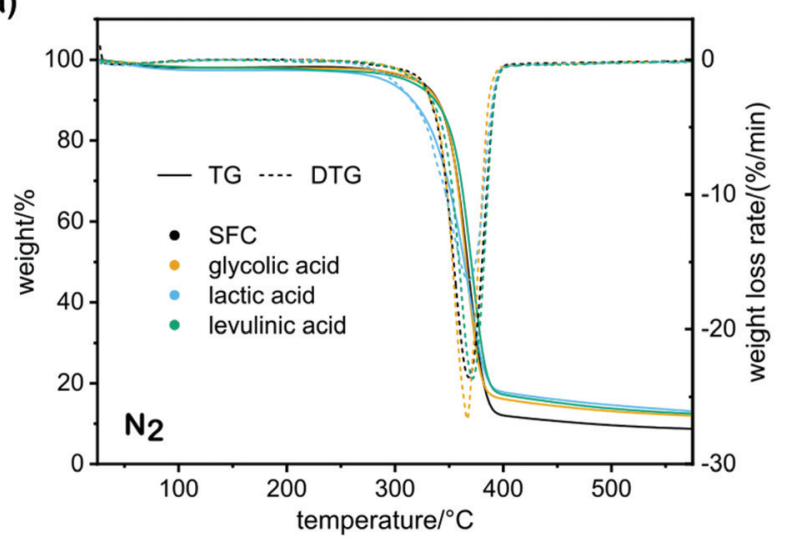

(b)

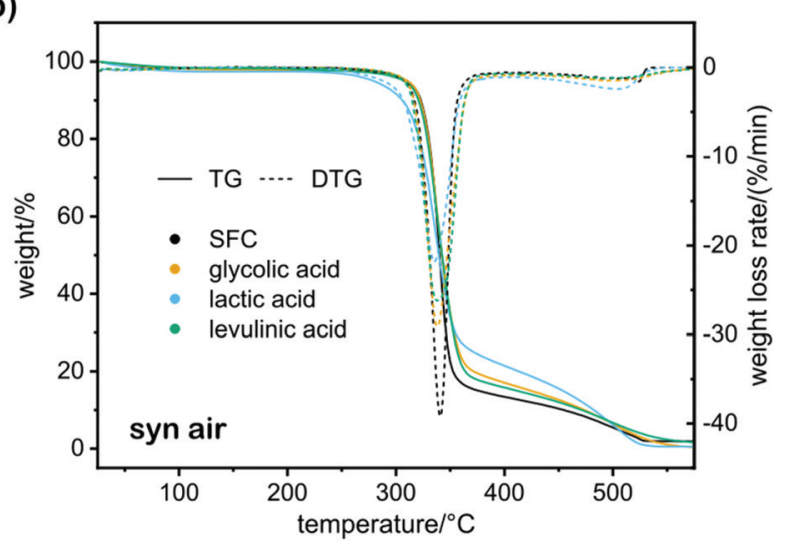

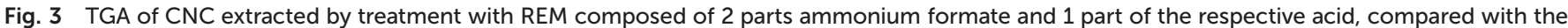
starting material SFC.

hydrolysis. ${ }^{30,36}$ However, the TGA of the starting material and the nanocellulose samples produced via the method proposed here do not show an onset degradation temperature significantly changed through the REM treatment (Fig. 3). Presumably, the amination of the reducing ends decreases their reductive activity, resulting in a material with higher thermal stability than previously reported carboxylated, ${ }^{37}$ carboxy-methylated, ${ }^{38}$ or phosphorylated ${ }^{39}$ CNCs.

Judging from the similarity of the product characteristics, the type of organic acid used as a component of the REM has little influence on the mechanism or success of the treatment. Further studies are therefore conducted using only the glycolic acid-containing REM. The reactivity of the REM towards carbohydrates is rather related to the unique combination of an organic acid with the Leuckart reagent ammonium formate. In the liquid state, ammonium formate exists in a Lewis acid-base dissociative equilibrium with ammonia and formic acid, influenced by the presence of the additional acidic REM component. For all the tested REM, heating over $120^{\circ} \mathrm{C}$ starts the intensive formation of ammonia, detected in the TGA-MS (Fig. 4) as frag- ments with $m / z=17,16,15$, revealing lower thermal stability of ammonium formate within the REM compared to the neat compound $\left(T_{\mathrm{d}}\left(\mathrm{HCOONH}_{4}\right) \approx 180^{\circ} \mathrm{C}\right)$. Thermal degradation of the REM already starts around $80^{\circ} \mathrm{C}$ with a significant loss of water $(m / z=18)$ and ammonia. Combined with the signals corresponding to formic acid $(\mathrm{m} / \mathrm{z}=46)$ and its fragments $(\mathrm{m} / \mathrm{z}=45$ : HCOO, $m / z=29$ : HCO), this implies the decomposition of ammonium formate into ammonia and formic acid as well as minor formation of formamide as a by-product. This in situ generated ammonia provides the unusual reactivity of REM towards the amination reaction. The formamide formation is accelerated at higher temperatures and can be monitored by the ion fragments $\mathrm{NH}_{2}-\mathrm{C}=\mathrm{O}^{+}(m / z=44)$ and $\mathrm{CH}_{2}=\mathrm{NH}_{2}{ }^{+}(m / z=30)$.

Considering that the thermal conversion of the REM starts at $80{ }^{\circ} \mathrm{C}$ already, and that the Leuckart reaction usually takes place around $140{ }^{\circ} \mathrm{C}-180^{\circ} \mathrm{C}$ (upper temperature is limited by the decomposition of the neat ammonium formate), the reaction temperature was decreased to as low as $140{ }^{\circ} \mathrm{C}$ and the reaction time was varied between $1 \mathrm{~h}$ and $6 \mathrm{~h}$. This also considers the maximum in the occurrence of the aminating
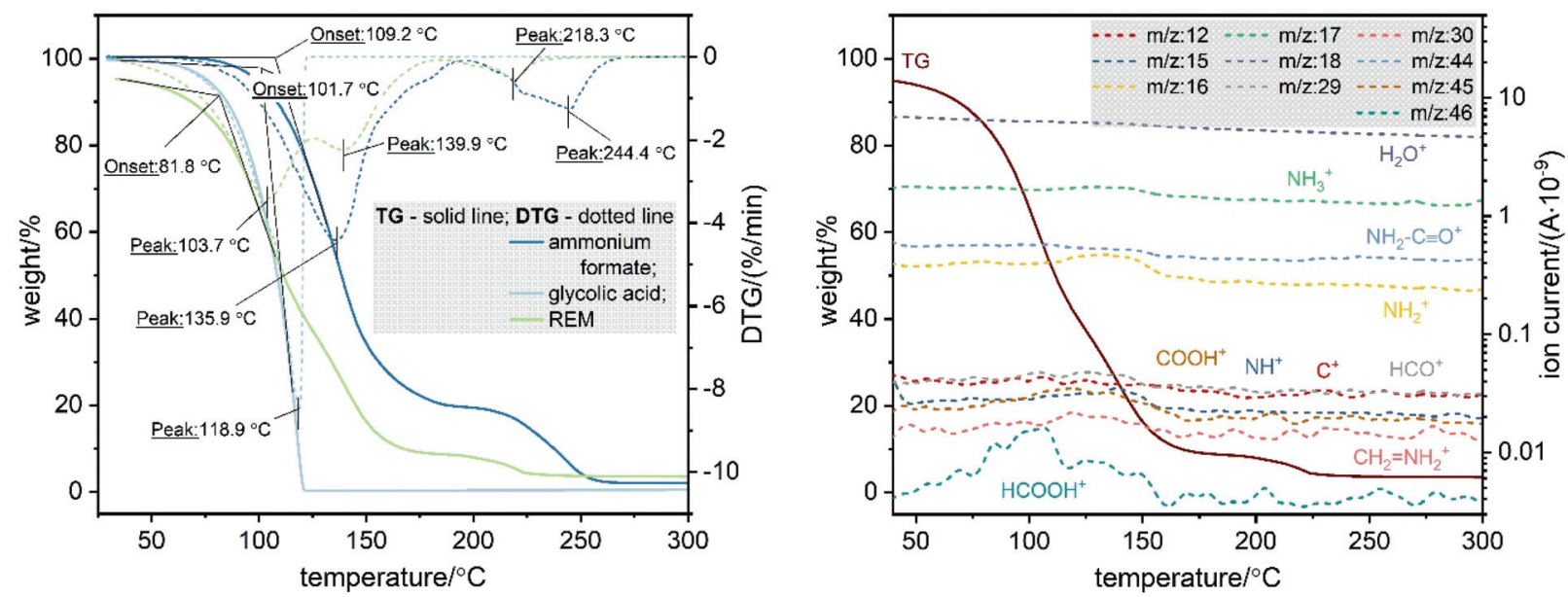

Fig. 4 Left: TGA of REM composed of ammonium formate and glycolic acid in 2:1 molar ratio. Right: TGA-MS of the same REM. 
(a)

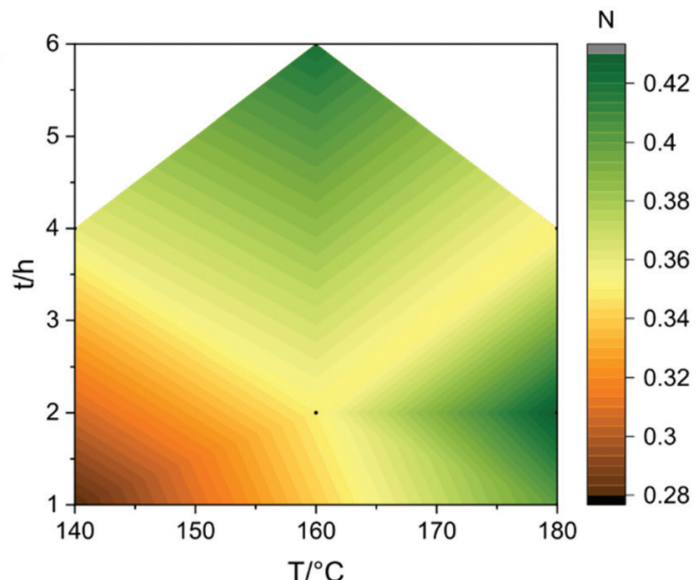

(b)

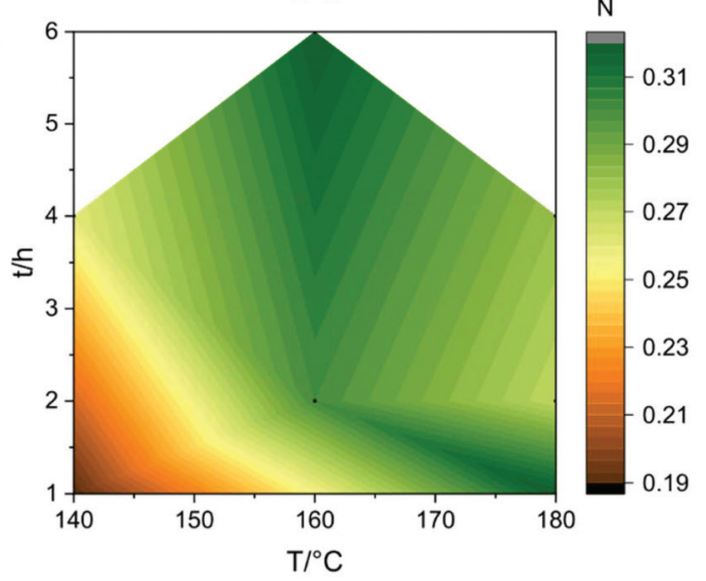

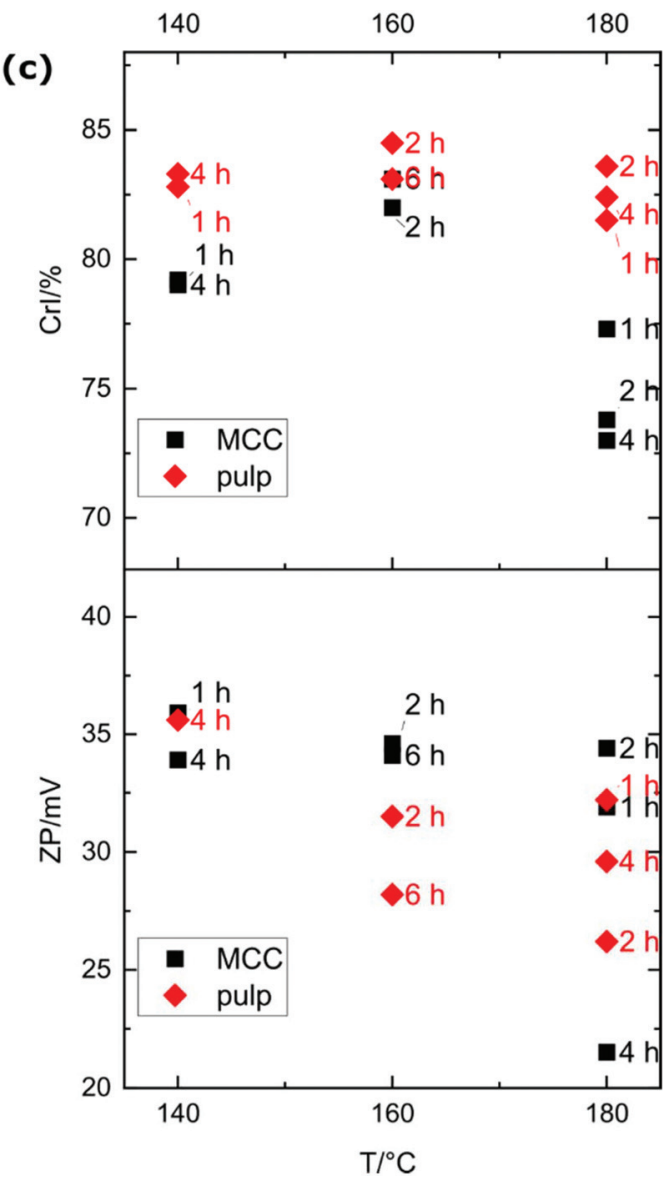

Fig. 5 (a) Nitrogen content of REM1-treated SFC samples; (b) nitrogen content of REM1-treated pulp samples dependent on reaction temperature and time; (c) zeta potential and crystallinity index of all samples dependent on the reaction temperature.

species, detected as $\mathrm{NH}_{3}{ }^{+}$and $\mathrm{NH}_{2}{ }^{+}$ions in TGA-MS, observed in the temperature range of $140-160{ }^{\circ} \mathrm{C}$. The CNC products exhibit an increase of both nitrogen content and CrI compared to the starting materials in every tested reaction condition (Fig. 5a-c; Table S1†). At milder reaction conditions, i.e. lower temperature and shorter reaction time, the amount of caramelization or Maillard by-products were visibly reduced, yielding a much brighter reaction mixture. Apparently, lower temperatures suffice to provide the activation energy for the aminolysis of amorphous cellulose, while the thermodynamically more stable crystalline domains remain intact and are extracted as the desired CNC. A higher reaction temperature of $180^{\circ} \mathrm{C}$ promotes aminolysis, i.e. depolymerization, of crystalline domains, demonstrated by a decreased CrI and broadening of the XRD maxima.

The variation between the zeta potential measured for samples treated for different times at the same temperature increases with increasing temperature (see Fig. 5c), suggesting a higher reproducibility at lower temperatures. In contrast, carrying out the reaction for a longer time at $180{ }^{\circ} \mathrm{C}$ results in partial decomposition of the material, $\mathrm{CNC}$ with poorer structural characteristics, and excessive formation of the nonenzymatic browning reaction by-products from soluble monosac- charides. The treatment at $160{ }^{\circ} \mathrm{C}$ results in the highest crystallinities, suggesting optimal conditions for the CNC extraction.

\section{Conclusions}

We have developed a sustainable method to extract cationically modified cellulose nanocrystals in an efficient one-step process using natural raw materials with low toxicity. The mechanism of the extraction appears to be based on a reductive amination, which proved to be most controllable and effective in a temperature range of $140-160{ }^{\circ} \mathrm{C}$. In these conditions, a balance is achieved between aminolysis of amorphous cellulose domains and preservation of the native crystalline structure, while successfully introducing positively charged amino groups into the chains, resulting in water-dispersible CNCs with good thermal stability.

\section{Conflicts of interest}

There are no conflicts of interest to declare. 


\section{Acknowledgements}

This work was financially supported by Max Planck Society and National Key Research. Authors are grateful to Michaela König for the ssNMR measurement and to Dr Oleksandr Selyshchev for recording and interpreting the XPS spectra. Open Access funding provided by the Max Planck Society.

\section{References}

1 D. Klemm, E. D. Cranston, D. Fischer, M. Gama, S. A. Kedzior, D. Kralisch, F. Kramer, T. Kondo, T. Lindström, S. Nietzsche, K. Petzold-Welcke and F. Rauchfuß, Nanocellulose as a natural source for groundbreaking applications in materials science: Today's state, Mater. Today, 2018, 21(7), 720-748.

2 B. B. Buchanan, W. Gruissem and R. L. Jones, in Biochemistry \& molecular biology of plants, John Wiley \& Sons Inc., Chichester, West Sussex; Hoboken, NJ, 2nd edn, 2015.

3 Y. Nishiyama, U.-J. Kim, D.-Y. Kim, K. S. Katsumata, R. P. May and P. Langan, Periodic Disorder along Ramie Cellulose Microfibrils, Biomacromolecules, 2003, 4(4), 10131017.

4 M. Antonietti, Sustainable Bulk Structural Material Engineered from Cellulose Nanofibers, Matter, 2020, 3(2), 339-340.

5 H. Yu, S. Y. H. Abdalkarim, H. Zhang, C. Wang and K. C. Tam, Simple Process To Produce High-Yield Cellulose Nanocrystals Using Recyclable Citric/Hydrochloric Acids, ACS Sustainable Chem. Eng., 2019, 7(5), 4912-4923.

6 T. Heinze, K. Schwikal and S. Barthel, Ionic liquids as reaction medium in cellulose functionalization, Macromol. Biosci., 2005, 5(6), 520-525.

7 A. Satlewal, R. Agrawal, S. Bhagia, J. Sangoro and A. J. Ragauskas, Natural deep eutectic solvents for lignocellulosic biomass pretreatment: Recent developments, challenges and novel opportunities, Biotechnol. Adv., 2018, 36(8), 2032-2050.

8 K. D. O. Vigier, G. Chatel and F. Jérôme, Contribution of Deep Eutectic Solvents for Biomass Processing: Opportunities, Challenges, and Limitations, ChemCatChem, 2015, 7(8), 1250-1260.

9 Q. Zhang, K. De Oliveira Vigier, S. Royer and F. Jerome, Deep eutectic solvents: syntheses, properties and applications, Chem. Soc. Rev., 2012, 41(21), 7108-7146.

10 P. Li, J. A. Sirvio, A. Haapala and H. Liimatainen, Cellulose Nanofibrils from Nonderivatizing Urea-Based Deep Eutectic Solvent Pretreatments, ACS Appl. Mater. Interfaces, 2017, 9(3), 2846-2855.

11 T. Suopajarvi, J. A. Sirvio and H. Liimatainen, Nanofibrillation of deep eutectic solvent-treated paper and board cellulose pulps, Carbohydr. Polym., 2017, 169, 167175.
12 O. Laitinen, T. Suopajärvi, M. Österberg and H. Liimatainen, Hydrophobic, Superabsorbing Aerogels from Choline Chloride-Based Deep Eutectic Solvent Pretreated and Silylated Cellulose Nanofibrils for Selective Oil Removal, ACS Appl. Mater. Interfaces, 2017, 9(29), 25029-25037.

13 P. Li, J. A. Sirviö, A. Haapala and H. Liimatainen, Cellulose Nanofibrils from Nonderivatizing Urea-Based Deep Eutectic Solvent Pretreatments, ACS Appl. Mater. Interfaces, 2017, 9(3), 2846-2855.

14 P. Li, J. A. Sirvio, B. Asante and H. Liimatainen, Recyclable deep eutectic solvent for the production of cationic nanocelluloses, Carbohydr. Polym., 2018, 199, 219-227.

15 O. Laitinen, J. Ojala, J. A. Sirviö and H. Liimatainen, Sustainable stabilization of oil in water emulsions by cellulose nanocrystals synthesized from deep eutectic solvents, Cellulose, 2017, 24(4), 1679-1689.

16 T. Selkälä, J. A. Sirviö, G. S. Lorite and H. Liimatainen, Anionically Stabilized Cellulose Nanofibrils through Succinylation Pretreatment in Urea-Lithium Chloride Deep Eutectic Solvent, ChemSusChem, 2016, 9(21), 3074-3083.

17 J. A. Sirviö, M. Visanko and H. Liimatainen, Acidic Deep Eutectic Solvents As Hydrolytic Media for Cellulose Nanocrystal Production, Biomacromolecules, 2016, 17(9), 3025-3032.

18 J. A. Sirviö and M. Visanko, Anionic wood nanofibers produced from unbleached mechanical pulp by highly efficient chemical modification, J. Mater. Chem. A, 2017, 5(41), 21828-21835.

19 J. A. Sirviö, J. Ukkola and H. Liimatainen, Direct sulfation of cellulose fibers using a reactive deep eutectic solvent to produce highly charged cellulose nanofibers, Cellulose, 2019, 26(4), 2303-2316.

20 P. Li, J. A. Sirviö, B. Asante and H. Liimatainen, Recyclable deep eutectic solvent for the production of cationic nanocelluloses, Carbohydr. Polym., 2018, 199, 219227.

21 J. A. Sirviö, Cationization of lignocellulosic fibers with betaine in deep eutectic solvent: Facile route to charge stabilized cellulose and wood nanofibers, Carbohydr. Polym., 2018, 198, 34-40.

22 H. Liimatainen, T. Suopajärvi, J. Sirviö, O. Hormi and J. Niinimäki, Fabrication of cationic cellulosic nanofibrils through aqueous quaternization pretreatment and their use in colloid aggregation, Carbohydr. Polym., 2014, 103, 187-192.

23 H. Sehaqui, A. Mautner, U. Perez de Larraya, N. Pfenninger, P. Tingaut and T. Zimmermann, Cationic cellulose nanofibers from waste pulp residues and their nitrate, fluoride, sulphate and phosphate adsorption properties, Carbohydr. Polym., 2016, 135, 334-340.

24 X.-M. Fan, H.-Y. Yu, D.-C. Wang, Z.-H. Mao, J. Yao and K. C. Tam, Facile and Green Synthesis of Carboxylated Cellulose Nanocrystals as Efficient Adsorbents in Wastewater Treatments, ACS Sustainable Chem. Eng., 2019, 7(21), 18067-18075. 
25 Z. Zhu, S. Fu, N. Lavoine and L. A. Lucia, Structural reconstruction strategies for the design of cellulose nanomaterials and aligned wood cellulose-based functional materials - A review, Carbohydr. Polym., 2020, 247, 116722.

26 Y. Markushyna, A. Volkel, A. Savateev, M. Antonietti and S. Filonenko, One-pot photocalalytic reductive formylation of nitroarenes via multielectron transfer by carbon nitride in functional eutectic medium, J. Catal., 2019, 380, 186194.

27 S. Filonenko, A. Voelkel and M. Antonietti, Valorization of monosaccharides towards fructopyrazines in a new sustainable and efficient eutectic medium, Green Chem., 2019, 21(19), 5256-5266.

28 Y. Habibi, L. A. Lucia and O. J. Rojas, Cellulose Nanocrystals: Chemistry, Self-Assembly, and Applications, Chem. Rev., 2010, 110(6), 3479-3500.

29 O. A. Battista, S. Coppick, J. A. Howsmon, F. F. Morehead and W. A. Sisson, Level-Off Degree of Polymerization, Ind. Eng. Chem., 1956, 48(2), 333-335.

30 O. M. Vanderfleet, M. S. Reid, J. Bras, L. Heux, J. GodoyVargas, M. K. R. Panga and E. D. Cranston, Insight into thermal stability of cellulose nanocrystals from new hydrolysis methods with acid blends, Cellulose, 2019, 26(1), 507528.

31 G. Socrates, Infrared and Raman characteristic group frequencies. Tables and charts George Socrates John Wiley and Sons, Ltd, Chichester, Third Edition, 2001, J. Raman Spectrosc., 2004, 35(10), 905-905.
32 P. J. Larkin, General Outline for IR and Raman Spectral Interpretation, in Infrared and Raman Spectroscopy (Second Edition), ed. P. J. Larkin, Elsevier, 2018, ch. 7, pp. 135-151.

33 P. B. Groszewicz, P. Mendes, B. Kumari, J. Lins, M. Biesalski, T. Gutmann and G. Buntkowsky, $\mathrm{N}$-Hydroxysuccinimide-activated, esters as a functionalization agent for amino cellulose: synthesis and solid-state NMR characterization, Cellulose, 2020, 27(3), 1239-1254.

34 A. Domard, C. Gey, M. Rinaudo and C. Terrassin, 13C and 1H n.m.r. spectroscopy of chitosan and N-trimethyl chloride derivatives, Int. J. Biol. Macromol., 1987, 9(4), 233-237.

35 A. Potthast, T. Rosenau and P. Kosma, Analysis of Oxidized Functionalities in Cellulose, in Polysaccharides II, ed. D. Klemm, Springer Berlin Heidelberg, Berlin, Heidelberg, 2006, pp. 1-48.

36 S. Matsuoka, H. Kawamoto and S. Saka, What is active cellulose in pyrolysis? An approach based on reactivity of cellulose reducing end, J. Anal. Appl. Pyrolysis, 2014, 106, 138-146.

37 H. Fukuzumi, T. Saito, Y. Okita and A. Isogai, Thermal stabilization of TEMPO-oxidized cellulose, Polym. Degrad. Stab., 2010, 95(9), 1502-1508.

38 D. de Britto and O. B. G. Assis, Thermal degradation of carboxymethylcellulose in different salty forms, Thermochim. Acta, 2009, 494(1), 115-122.

39 M. Ghanadpour, F. Carosio, P. T. Larsson and L. Wågberg, Phosphorylated Cellulose Nanofibrils: A Renewable Nanomaterial for the Preparation of Intrinsically FlameRetardant Materials, Biomacromolecules, 2015, 16(10), 3399-3410. 